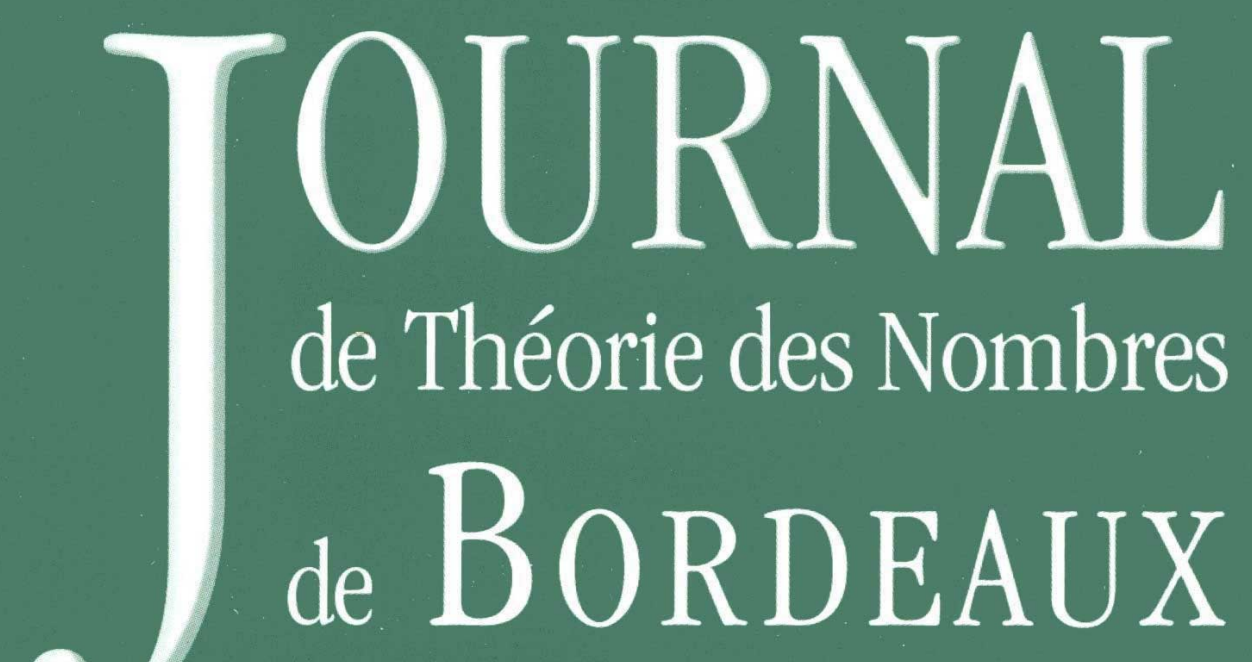

anciennement Séminaire de Théorie des Nombres de Bordeaux

\title{
Ritabrata MUNSHI
}

Density of rational points on cyclic covers of $\mathbb{P}^{n}$

Tome 21, no 2 (2009), p. 335-341.

<http://jtnb.cedram.org/item?id=JTNB_2009__21_2_335_0>

(C) Université Bordeaux 1, 2009, tous droits réservés.

L'accès aux articles de la revue «Journal de Théorie des Nombres de Bordeaux » (http://jtnb.cedram.org/), implique l'accord avec les conditions générales d'utilisation (http://jtnb.cedram. org/legal/). Toute reproduction en tout ou partie cet article sous quelque forme que ce soit pour tout usage autre que l'utilisation à fin strictement personnelle du copiste est constitutive d'une infraction pénale. Toute copie ou impression de ce fichier doit contenir la présente mention de copyright.

\section{cedram}




\title{
Density of rational points on cyclic covers of $\mathbb{P}^{n}$
}

\author{
par RITABRATA MUNSHI
}

RÉSUMÉ. Nous obtenons une majoration de la densité des points rationnels sur les revêtements cycliques de $\mathbb{P}^{n}$. Quand $n \rightarrow \infty$ notre estimation tend vers la majoration conjecturale de Serre.

ABSTRACT. We obtain upper bound for the density of rational points on the cyclic covers of $\mathbb{P}^{n}$. As $n \rightarrow \infty$ our estimate tends to the conjectural bound of Serre.

\section{Introduction}

Let $\mathbb{P}^{n}$ denote the $n$-dimensional projective space over the field of rational numbers. For a finite cover $f: X \rightarrow \mathbb{P}^{n}$ over $\mathbb{Q}$, one can define a counting function

$$
N(f, B)=|\{P \in X(\mathbb{Q}): H(f(P)) \leq B\}|,
$$

where $H$ is the standard multiplicative height function defined on $\mathbb{P}^{n}$. If the degree of $f$ is at least two, Serre [10] proved that

$$
N(f, B) \ll B^{n+\frac{1}{2}}(\log B)^{\gamma}
$$

with $\gamma<1$. Here the implied constant depends both on $f$ and $\gamma$. Serre's proof is based on an analogous result proved by Cohen [3] in the affine case. The proof is a beautiful application of the large sieve inequalities. Serre [10] also conjectures that one should have

$$
N(f, B) \ll B^{n}(\log B)^{c},
$$

for some $c$. Broberg [2] almost settled Serre's conjecture for covers of the projective line and the projective plane. More precisely, he proved:

(1) If $f: X \rightarrow \mathbb{P}^{1}$ is a cover of degree $d$, then for every $\varepsilon>0$ we have

$$
N(f, B) \ll_{\epsilon, f} B^{\frac{2}{d}+\varepsilon} .
$$

(2) If $f: X \rightarrow \mathbb{P}^{2}$ is a cover of degree $d>2$, then for every $\varepsilon>0$ we have

$$
N(f, B) \ll_{\epsilon, f} B^{2+\varepsilon} .
$$

In the case $d=2$, we have

$$
N(f, B) \ll_{\epsilon, f} B^{\frac{9}{4}+\varepsilon}
$$

for every $\varepsilon>0$. 
Broberg's method is based on that introduced by Heath-Brown in [7]. These methods, though elementary, have proved to be powerful in many situations. In fact the last result of Broberg for degree two covers of $\mathbb{P}^{2}$, is the best known result towards Manin's conjecture, which predicts the exponent to be $1+\varepsilon$, for del Pezzo surfaces of degree 2. Here we will give a slightly better estimate albeit at the cost of employing heavy machinery.

To this end let $F\left(x_{0}, \ldots, x_{n}\right)$ be an irreducible homogenous polynomial of degree $m d$, with $d>1$. Moreover suppose that the projective hypersurface defined by $F(\mathbf{x})=0$ is smooth. Then the equation

$$
y^{d}=F\left(x_{0}, \ldots, x_{n}\right)
$$

defines a variety $X$ in the weighted projective space $\mathbb{P}(m, 1, \ldots, 1)$, where $y$ is given weight $m$ and each $x_{i}$ is given weight 1 . This variety can be viewed as a cyclic $d$-sheeted cover of the projective space $\mathbb{P}^{n}$, via the natural map

$$
\begin{aligned}
f: X & \rightarrow \mathbb{P}^{n} ; \\
\left(y, x_{0}, \ldots, x_{n}\right) & \mapsto\left(x_{0}, \ldots, x_{n}\right) .
\end{aligned}
$$

This cover is ramified above the hypersurface $F(\mathbf{x})=0$. Using adjunction formula one easily verifies the canonical sheaf of $X$ to be

$$
\omega_{X}=O_{X}(-m+m d-n-1) .
$$

Hence, if the parameters $n, m$ and $d$ are such that

$$
m d-m<n+1
$$

then the variety $X$ is a Fano variety, i.e. the anticanonical sheaf is ample. In this case we have a more precise conjecture due to Manin et al ([1], [5]), regarding the density of rational points.

In this paper, we employ the power sieve together with Poisson summation formula, and deep results of Deligne [4], and of Katz [9] about cancellation in mixed character sums over finite fields, to estimate the number of $d$-powers in the set

$$
\left\{F\left(x_{0}, \ldots, x_{n}\right):-B<x_{i}<B\right\}
$$

with some smooth weight $W$. This will give us our main result.

Theorem 1.1. Let $f: X \rightarrow \mathbb{P}^{n}$ be a finite cyclic cover of $\mathbb{P}^{n}$ over $\mathbb{Q}$. Then we have

$$
N(f, B) \ll_{f} B^{n+\frac{1}{n+2}}(\log B)^{\frac{n+1}{n+2}} .
$$

Remark. In the special case of cyclic covers our result is better than that of Serre [10]. Also it is exciting to note that as the dimension $n$ gets larger our bound comes closer to the conjectured bound of Serre. 
Remark. For $n=2$ and $d=2$ our result gives

$$
N(f, B) \ll B^{\frac{9}{4}}(\log B)^{\frac{3}{4}},
$$

which improves the result of Broberg by an $\varepsilon$. However, for $n=2, d>2$ our result is worse.

\section{Power sieve}

In this section we briefly recall the concept of $d$-power sieve (see [6] for $d=2)$. Let $p$ be a prime such that $p \equiv 1(\bmod d)$. Then for every non-zero element $a$ in the finite field $\mathbb{F}_{p}$, we can define a $d$-th root of unity by $a^{\frac{p-1}{d}}$. Now since $\mathbb{F}_{p}^{*}$ is cyclic, we can define a non-canonical isomorphism

$$
\theta_{p}: \mathbb{F}_{p}^{*} \rightarrow \mu_{p-1}
$$

where $\mu_{p-1}$ denotes the group of $(p-1)$-th roots of unity in $\mathbb{C}^{*}$. Using this we can define a primitive Dirichlet character modulo $p$,

$$
\chi_{p}(n)=\theta_{p}\left(\bar{n}^{\frac{p-1}{d}}\right)
$$

for $(p, n)=1$, and $\chi_{p}(n)=0$ for $p \mid n$. The crucial property of this character is the following:

$$
\chi_{p}(n)=1 \text { if }(p, n)=1 \text {, and } n=m^{d} \text { for some } m .
$$

Now let $\mathcal{A}=(a(n))$ be a finite sequence of non-negative quantities. We are interested in estimating the sum over the $d$-powers

$$
S(\mathcal{A})=\sum_{n} a\left(n^{d}\right)
$$

Let $\mathcal{P}$ be a set of $P$ primes each having residue 1 modulo $d$. Suppose that $a(n)=0$ if $|n| \geq e^{P}$, and consider the expression

$$
S=\sum_{n} a(n)\left|\sum_{p \in \mathcal{P}} \chi_{p}(n)\right|^{2}
$$

Each $n$ is counted with a non-negative weight, and if $n=m^{d}$ and $0<|n|<$ $e^{P}$ then

$$
\sum_{p \in \mathcal{P}} \chi_{p}(n)=\sum_{\substack{p \in \mathcal{P} \\(p, n)=1}} 1 \gg P .
$$

Hence $P^{2} S(\mathcal{A}) \ll S$. Moreover

$$
\begin{aligned}
S & =\sum_{p, q \in \mathcal{P}} \sum_{n} a(n) \chi_{p}(n) \bar{\chi}_{q}(n) \\
& \leq P \sum_{n} a(n)+\sum_{p \neq q \in \mathcal{P}}\left|\sum_{n} a(n) \chi_{p}(n) \bar{\chi}_{q}(n)\right| .
\end{aligned}
$$

Hence we obtain the following: 
Lemma 2.1. Let $\mathcal{P}$ be a set of $P$ primes each having residue 1 modulo $d$. Suppose that $a(n)=0$ for $n=0$ and for $|n| \geq e^{P}$. Then

$$
S(\mathcal{A}) \ll P^{-1} \sum_{n} a(n)+P^{-2} \sum_{p \neq q \in \mathcal{P}}\left|\sum_{n} a(n) \chi_{p}(n) \bar{\chi}_{q}(n)\right| .
$$

\section{Proof of Theorem 1}

Let $W: \mathbb{R}^{n+1} \rightarrow \mathbb{R}$ be a non-negative smooth function supported in the dyadic box $[-B, B]^{n+1}$, and such that the partial derivatives satisfy the following bound:

$$
\left|\frac{d^{i_{0}+\cdots+i_{n}} W\left(x_{0}, \ldots, x_{n}\right)}{d x_{0}^{i_{0}} \ldots d x_{n}^{i_{n}}}\right| \ll B^{-\left(i_{0}+\cdots+i_{n}\right)} .
$$

Then via integration-by-parts we note the following bound for the Fourier transform

$$
\hat{W}(\mathbf{u})=\int_{\mathbb{R}^{n+1}} W(\mathbf{x}) e(-\langle\mathbf{x}, \mathbf{u}\rangle) d \mathbf{x} \ll B^{n+1} \prod_{i=0}^{n}\left(1+\left|u_{i}\right| B\right)^{-2}
$$

where $\mathbf{u}=\left(u_{0}, \ldots, u_{n}\right)$.

Then we define a sequence of non-negative numbers by

$$
a(k)=\sum_{\mathbf{x} \in \mathbb{Z}^{n+1}, F(\mathbf{x})=k} W(\mathbf{x}) .
$$

We wish to obtain an upper bound for

$$
S(\mathcal{A})=\sum_{k} a\left(k^{d}\right)
$$

using the $d$-power sieve. Now clearly we have

$$
\sum_{k} a(k) \ll B^{n+1} \text {. }
$$

Hence to apply the lemma we need to estimate the sum

$$
S(p, q)=\sum_{k} a(k) \chi_{p}(k) \bar{\chi}_{q}(k)=\sum_{\mathbf{x} \in \mathbb{Z}^{n+1}} W(\mathbf{x}) \chi_{p}(F(\mathbf{x})) \bar{\chi}_{q}(F(\mathbf{x})),
$$

where $p$ and $q$ are two distinct primes. Using Poisson summation formula we obtain

$$
\begin{aligned}
S(p, q) & =\sum_{\mathbf{a}(\bmod p q)} \chi_{p}(F(\mathbf{a})) \bar{\chi}_{q}(F(\mathbf{a})) \sum_{\mathbf{x} \equiv \mathbf{a}} W(\mathbf{x}) \\
& =(p q)^{-(n+1)} \sum_{\mathbf{u} \in \mathbb{Z}^{n+1}} g(\mathbf{u} ; p, q) \hat{W}\left(\frac{\mathbf{u}}{p q}\right)
\end{aligned}
$$


where

$$
g(\mathbf{u} ; p, q)=\sum_{\mathbf{a}(\bmod p q)} \chi_{p}(F(\mathbf{a})) \bar{\chi}_{q}(F(\mathbf{a})) e\left(\frac{\langle\mathbf{a}, \mathbf{u}\rangle}{p q}\right)
$$

is a mixed character sum. Then we observe that the above sum satisfies the following multiplicative property

$$
g(\mathbf{u} ; p, q)=g\left(\bar{q} \mathbf{u} ; \chi_{p}\right) g\left(\bar{p} \mathbf{u} ; \bar{\chi}_{q}\right)
$$

where $p \bar{p} \equiv 1(\bmod q), q \bar{q} \equiv 1(\bmod p)$ and

$$
g\left(\mathbf{u} ; \chi_{p}\right)=\sum_{\mathbf{a}(\bmod p)} \chi_{p}(F(\mathbf{a})) e\left(\frac{\langle\mathbf{a}, \mathbf{u}\rangle}{p}\right) .
$$

So our job is reduced to estimating the mixed character sum over the finite field $\mathbb{F}_{p}$. Let $V$ be the hypersurface defined by $F(\mathbf{x})=0$ over the field of rationals. Let $V^{*}$ be its dual variety, which in this case is again a hypersurface. Assuming that the prime $p$ is such that the reduction $V_{p}=$ $V(\bmod p)$ is smooth, we have three situations:

(1) First suppose that the vector $\mathbf{u} \in \mathbb{Z}^{n+1}$ is such that $\mathbf{u} \equiv \mathbf{0}(\bmod p)$, then

$$
g\left(\mathbf{u} ; \chi_{p}\right)=\sum_{\mathbf{a}(\bmod p)} \chi_{p}(F(\mathbf{a})) \ll p^{\frac{n+1}{2}},
$$

where the implied constant depends only on $n$ and the degree of $F$. This follows from the multiplicative analogue of Deligne's bound established by Katz [8].

(2) If $\mathbf{u}(\bmod p)$ is non-zero and the associated hyperplane $\langle\mathbf{u}, \mathbf{x}\rangle=0$ is not a tangent to the hypersurface $V_{p}$, then by Theorem 1.1 in [9] we have square-root cancellation i.e.

$$
g\left(\mathbf{u} ; \chi_{p}\right) \ll p^{\frac{n+1}{2}} .
$$

Here again the implied constant depends only on $n$ and the degree of $F$. In particular, it does not depend on $\mathbf{u}$.

(3) If $\mathbf{u}(\bmod p)$ is non-zero and the associated hyperplane $\langle\mathbf{u}, \mathbf{x}\rangle=0$ is a tangent to the hypersurface $V_{p}$, then using the primitivity of the character we get

$$
g\left(\mathbf{u} ; \chi_{p}\right)=\frac{\chi_{p}(-1) \tau\left(\chi_{p}\right)}{p} \sum_{\mathbf{b}(\bmod p)} \bar{\chi}_{p}(\mathbf{b}) \sum_{\mathbf{a}(\bmod p)} e\left(\frac{\mathbf{b} F(\mathbf{a})+\langle\mathbf{a}, \mathbf{u}\rangle}{p}\right),
$$

where $\tau(\chi)$ stands for the Gauss sum of the character $\chi$. Then we apply Deligne's bound [4] to the inner sum to obtain

$$
g\left(\mathbf{u} ; \chi_{p}\right) \ll \sqrt{p} p^{\frac{n+1}{2}} .
$$

Again the implied constant depends only on $n$ and the degree of $F$. 
In the first two cases we say that the vector $\mathbf{u} \in \mathbb{Z}^{n+1}$ is 'good' modulo $p$. In the third case we say that $\mathbf{u}$ is 'bad' modulo $p$.

We first compute the contribution to $S(p, q)$ from those vectors $\mathbf{u} \in \mathbb{Z}^{n+1}$ which are 'good' modulo both $p$ and $q$. Using square-root cancellation we see that this is bounded by

$$
\ll B^{n+1} \frac{(p q)^{\frac{n+1}{2}}}{(p q)^{n+1}} \sum_{\mathbf{u} \in \mathbb{Z}^{n+1}} \prod_{i=0}^{n}\left(1+\frac{\left|u_{i}\right| B}{p q}\right)^{-2} \ll(p q)^{\frac{n+1}{2}} .
$$

In the last inequality we are assuming that $p q \geq B$, which follows from our choice of the set $\mathcal{P}$. Now the contribution of those vectors $\mathbf{u} \in \mathbb{Z}^{n+1}$ which are 'bad' for the prime $p$ but 'good' for the prime $q$ is given by

$$
(p q)^{-(n+1)} \sum_{\mathbf{v} \in V_{p}^{*}\left(\mathbb{F}_{p}\right)} \sum_{\lambda=1}^{p} \sum_{\mathbf{u} \equiv \lambda \mathbf{v}(\bmod p)}^{*} g(\mathbf{u} ; p, q) \hat{W}\left(\frac{\mathbf{u}}{p q}\right) .
$$

The $*$ above the sum indicates that the sum is restricted over those vectors $\mathbf{u} \in \mathbb{Z}^{n+1}$ which are 'good' modulo $q$. The above expression is bounded by

$$
\begin{aligned}
& B^{n+1} \frac{p^{\frac{n+2}{2}} q^{\frac{n+1}{2}}}{(p q)^{n+1}} \sum_{\mathbf{v} \in V_{p}^{*}\left(\mathbb{F}_{p}\right)} \sum_{\lambda=1}^{p} \sum_{\mathbf{u} \equiv \lambda \mathbf{v}(\bmod p)}^{*} \prod_{i=0}^{n}\left(1+\frac{\left|u_{i}\right| B}{p q}\right)^{-2} \\
\ll & B^{n+1} \frac{p^{\frac{n+2}{2}} q^{\frac{n+1}{2}}}{(p q)^{n+1}} \sum_{\mathbf{v} \in V_{p}^{*}\left(\mathbb{F}_{p}\right)} \sum_{\lambda=1}^{p}\left(\frac{q}{B}\right)^{n+1} \ll p^{\frac{n}{2}} q^{\frac{n+1}{2}} .
\end{aligned}
$$

In the last inequality we have used the fact that $\left|V_{p}^{*}\left(\mathbb{F}_{p}\right)\right| \ll p^{n-1}$. Similarly one can show that the contribution of those vectors $\mathbf{u} \in \mathbb{Z}^{n+1}$ which are 'bad' for both $p$ and $q$, is bounded by $(p q)^{\frac{n}{2}}$. Hence we conclude that

$$
S(p, q) \ll(p q)^{\frac{n+1}{2}} .
$$

Now for the set $\mathcal{P}$ we pick $P$ consecutive primes in the progression $(1+$ $d k)_{k}$ of size $\sim P \log P$. Then using the $d$-power sieve inequality, we get

$$
S(\mathcal{A}) \ll \frac{B^{n+1}}{P}+(P \log P)^{n+1} .
$$

Then choosing

$$
P=\left(\frac{B}{\log B}\right)^{\frac{n+1}{n+2}}
$$

the theorem follows.

\section{Acknowledgements}

I thank the organizers of the 25th Journées Arithmétiques for inviting me to this exciting conference, and for the support. 


\section{References}

[1] V.V. Batyrev; Y.I. Manin, Sur le nombre des points rationnels de hauteur borné des variétés algébriques. Math. Ann. 286 (1990), 27-43.

[2] N. Broberg, Rational points on finite covers of $\mathbb{P}^{1}$ and $\mathbb{P}^{2}$. J. Number Theory 101 (2003), 195-207.

[3] S.D. Cohen, The distribution of Galois groups and Hilbert's irreducibility theorem. Proc. London Math. Soc. (3) 43 (1981), 227-250.

[4] P. Deligne, La conjecture de Weil. I. Inst. Hautes Études Sci. Publ. Math. No. 43 (1974), $273-307$.

[5] J. Franke; Y.I. Manin; Y. Tschinkel, Rational points of bounded height on Fano varieties. Invent. Math. 95 (1989), 421-435.

[6] D.R. Heath-Brown, The square sieve and consecutive square-free numbers. Math. Ann. 266 (1984), 251-259.

[7] D.R. Heath-Brown, The density of rational points on curves and surfaces. Ann. of Math. (2) 155 (2002), 553-595.

[8] N. KATz, Estimates for nonsingular multiplicative character sums. Int. Math. Res. Not. (2002), 333-349.

[9] N. KATZ, Estimates for nonsingular mixed character sums. Int. Math. Res. Not. (2007), vol. 2007, article ID rnm069, 19 pages, doi:10.1093/imrn/rnm069.

[10] J-P. Serre, Lectures on the Mordell-Weil theorem. Friedr. Vieweg \& Sohn, Braunschweig (1989).

Ritabrata Munshi

Rutgers University

110, Frelinghuysen Road

Piscataway NJ 08854, USA

E-mail: rmunshi@math.rutgers.edu

$U R L:$ http://www . math.rutgers.edu/ ${ }^{\sim}$ rmunshi 